\title{
INEQUALITIES FOR CERTAIN CYCLIC SUMS
}

\author{
by DAVID E. DAYKIN
}

(Received 30th May 1970)

\section{Introduction}

Let $M$ be a positive integer, let $a_{1}, a_{2}, \ldots, a_{M}$ be non-negative reals, and put $a_{M+i}=a_{i}$ for $i=1,2,3$. Further let each of $v_{1}, v_{2}, v_{3}$ and $\delta_{1}, \delta_{2}, \delta_{3}$ be 0 or 1 , giving $2^{6}$ possibilities. This note is concerned with the problem of finding bounds for each of the non-trivial cases out of the $2^{6}$ cyclic sums

$$
S_{M}=\sum_{i=1}^{M} \frac{v_{1} a_{i+1}+v_{2} a_{i+2}+v_{3} a_{i+3}}{\delta_{1} a_{i+1}+\delta_{2} a_{i+2}+\delta_{3} a_{i+3}} \text {. }
$$

Of course we do not allow zero denominators. Known results (1), (5) are

Theorem 1. If $\delta_{1}+\delta_{2}+\delta_{3}=1$ then $\left(v_{1}+v_{2}+v_{3}\right) M \leqq S_{M}<\infty$.

Theorem 2. If $v_{1}=\delta_{1}=\delta_{2}=1$ and $v_{3}=0$ then

$$
1+v_{2} \leqq S_{M} \leqq M-1+v_{2}-\delta_{3} \text {. }
$$

To simplify the notation let

$$
\Sigma \frac{a+c}{a+b} \text { mean } \sum_{i=1}^{M} \frac{a_{i+1}+a_{i+3}}{a_{i+1}+a_{i+2}}
$$

and so on. Then all non-trivial cases of (1), not covered by Theorems 1 and 2 , can be dealt with by means of one of the following:

$$
\begin{aligned}
& 0 \cdot 461238 M \leqq A=\Sigma \frac{a}{b+c}<\infty, \\
& M \leqq B=\Sigma \frac{a+b}{b+c}<\infty, \\
& \min \left\{2, \frac{1}{2} M\right\} \leqq C=\Sigma \frac{b}{a+c}<\infty, \\
& 1 \leqq D=\Sigma \frac{b}{a+b+c} \leqq\left[\frac{1}{2} M\right] \text { for } 3 \leqq M, \\
& \min \{4, M\} \leqq E=\Sigma \frac{a+b}{a+c}<\infty, \\
& {\left[\frac{1}{2}(M+1)\right] \leqq F=\Sigma \frac{a+c}{a+b}<\infty .}
\end{aligned}
$$

E.M.S. $-\mathbf{R}$ 
The most famous case of (1) is the sum $A$ in (2) above. Clearly $A_{M}=\frac{1}{2} M$ when all $a_{i}$ are equal, so $\inf A_{M} \leqq \frac{1}{2} M$. It was Shapiro who suggested the verification that inf $A_{M}=\frac{1}{2} M$. Diananda (3) proved that

$$
\inf A_{M+2} \leqq 1+\inf A_{M} \text {, }
$$

and that if $M$ is odd then $\inf A_{M+1} \leqq \frac{1}{2}+\inf A_{M}$. It has been shown by examples of $a_{i}$ that inf $A_{M}<\frac{1}{2} M$ for $M=14$ by Lighthill and Zulauf, and for $M=25$ by the author. On the other hand Nowosad (4) proved that

$$
\text { inf } A_{M}=\frac{1}{2} M \text { for } M=10 \text {. }
$$

Thus the only cases for which it is still not known whether inf $A_{M}<\frac{1}{2} M$ are $M=12$ and $11 \leqq M$ odd $\leqq 23$. The lower bound in (2) was obtained by Diananda (2), by developing a method of Rankin. However, the infimum of $A$ and $F$ are not known, and they seem to be hard to find. The other bounds given above are best possible, the infinite ones are trivial and will not be mentioned further.

It is easy (1) to prove (3). The four remaining inequalities (4)-(7) appear to be new and will be proved below. It will be shown that inf $F_{M}$ behaves in the same way as inf $A_{M}$, which we just described. Other new inequalities presented here are

$$
\begin{aligned}
& -M \varepsilon^{-1} \leqq G_{M}=\Sigma\left(\frac{a}{a+b}\right) \log \left(\frac{a}{a+b}\right) \leqq 0, \\
& -M \varepsilon^{-1} \leqq H_{M}=\Sigma\left(\frac{2 a}{a+b}\right) \log \left(\frac{2 a}{a+b}\right) \leqq 2(M-1) \log 2,
\end{aligned}
$$

where $\varepsilon>1$ is the base of the logarithms. Again inf $G_{M}$ and inf $H_{M}$ are not known, but it will be shown that they are both near $-M \varepsilon^{-1}$.

One can fairly easily obtain the corresponding results for $\Sigma t \log t$ when $t$ has any one of the following forms

$$
\begin{gathered}
\frac{a}{a+b+c}, \frac{3 a}{a+b+c}, \frac{a+b}{a+b+c}, \frac{3(a+b)}{2(a+b+c)} \\
\frac{b}{a+c}, \frac{2 b}{a+c}, \frac{b}{a+b+c}, \frac{3 b}{a+b+c}, \frac{a+b}{a+c}
\end{gathered}
$$

Here are some conjectures:

$$
\begin{aligned}
0 & \leqq \Sigma\left(\frac{2 a}{b+c}\right) \log \left(\frac{2 a}{b+c}\right) \\
\frac{1}{2} M \leqq \Phi(x) & =\Sigma\left(\frac{2 a}{b+c}\right)^{x} \text { provided } a_{i}>0, \text { for any real } x, \\
0 & \leqq \Sigma\left(\frac{a+c}{a+b}\right) \log \left(\frac{a+c}{a+b}\right) \\
M & \leqq \Sigma\left(\frac{a+c}{a+b}\right)^{2}
\end{aligned}
$$


Conjecture (10) is the logarithmic analogue of Shapiro's conjecture, and the fact that Shapiro's conjecture turned out to be wrong makes it necessary to say something about (10). In (1) the function $\Phi(x)$ in (11) was discussed, and it was shown that $\frac{\partial \Phi(0)}{\partial x} \leqq 0$ and $\Phi(0)=M$ and $\Phi(2) \geqq M$. Hence because $\Phi(x)$ is a convex function of $x$, for any given $a_{i}$, the minimum value of $\Phi(x)$ occurs with $0 \leqq x \leqq 2$. In fact computer studies of $\Phi(x)$ indicate that the minimum is near $x=0$, and they gave rise to conjecture (11). We can get $\Phi(x)$ as close as we like to $\frac{1}{2} M$ for $M$ even by letting $\left\{a_{i}\right\}=\delta, 1, \delta, 1, \ldots$ when $\delta$ and $x$ are both small. Since $\Phi(x)$ is convex and $\Phi(0)=M$, if a particular set of $a_{i}$ were to make (10) false then they would make $\frac{1}{2} \Phi(1)=A_{M}<\frac{1}{2} M$, and this is impossible for $M \leqq 10$ by Nowosad's theorem, so this proves (10) is true for $M \leqq 10$ and $a_{i}>0$. The proof is easier for $a_{i} \geqq 0$ and it seems that (11) holds also in this case. Replacing $A$ by $F$ in all this gives a corresponding set of conjectures, the most interesting ones perhaps are (12) and (13), both of which have been proved true for $M \leqq 4$ and tested for higher $M$ on a computer. Inequality (6) shows why we don't have something for $F$ corresponding to (11).

\section{Proof of Inequality (4)}

The cases $M=1,2,4$ are easy, and $\frac{3}{2} \leqq A_{3}=C_{3}$. We now use induction on $M$. Let $4<M$ and, without loss of generality, assume $a_{M}$ has the smallest value out of the $a_{i}$. Then

$$
\begin{aligned}
0 \leqq C_{M} & -C_{M-1}=C_{M}\left(a_{1}, a_{2}, \ldots, a_{M}\right)-C_{M-1}\left(a_{1}, a_{2}, \ldots, a_{M-1}\right) \\
= & \left\{\frac{a_{M-1}}{a_{M-2}+a_{M}}-\frac{a_{M-1}}{a_{M-2}+a_{1}}\right\}+\left\{\frac{a_{M}}{a_{M-1}+a_{1}}\right\}+\left\{\frac{a_{1}}{a_{M}+a_{2}}-\frac{a_{1}}{a_{M-1}+a_{2}}\right\}
\end{aligned}
$$

because $0 \leqq\{\ldots\}$ for each of the three brackets. Thus $2 \leqq \inf C_{M-1} \leqq \inf C_{M}$. To see that 2 is the best lower bound let $\left\{a_{i}\right\}=\lambda^{1}, \lambda^{2}, \lambda^{3}, \ldots, \lambda^{M-1}, \lambda^{M-1}$, where $\lambda$ is large.

\section{Proof of Inequality (5)}

First we deal with the left hand bound. To attain it let $a_{i}=\lambda^{i}$ for $1 \leqq i \leqq M$ where $\lambda$ is large. The case $M=3$ is trivial. That $\inf D_{M-1} \leqq \inf D_{M}$ when $3<M$ is proved in exactly the same way as for $C_{M}$ in section 2 above, and the result follows inductively.

To attain the upper bound $\left[\frac{1}{2} M\right]$ let $\left\{a_{i}\right\}=0,1,0,1, \ldots$. Next note that $1 \leqq$ the sum of any two adjacent terms of $D_{M}$. This establishes the inequality for $M$ even. Suppose $5 \leqq M$ odd and write $a, b, c, \ldots$ for $a_{1}, a_{2}, a_{3}, \ldots$. Then

$$
\begin{aligned}
\frac{\partial D}{\partial c}=-\frac{b}{(a+b+c)^{2}}+\frac{b+d}{(b+c+d)^{2}}-\frac{d}{(c+d+e)^{2}} & \begin{aligned}
\leqq 0 \text { if } a \leqq d \text { and } e \leqq b, \\
\geqq 0 \text { if } a \geqq d \text { and } e \geqq b .
\end{aligned}
\end{aligned}
$$


If condition (i) holds then $D$ increases as $c \rightarrow 0$. When $c=0$ one term of $D$ is zero, and summing the remaining pairs of adjacent terms shows that $D \leqq\left[\frac{1}{2} M\right]$. If condition (ii) holds then $D$ increases as $c \rightarrow \infty$, and in the limit we again have at least one zero term in $D$. The result now follows because one of conditions (i) and (ii) must hold for some renumbering of the $a_{i}$ 's. The renumbering must of course preserve the cycle of the $a_{i}$ 's.

\section{Proof of Inequality (6)}

We again use induction on $M$. The cases $M \leqq 4$ are easy, so suppose $5 \leqq M$ and that $a_{M}$ is the smallest $a_{i}$. Then

where

$$
E_{M}-E_{M-1}=t_{1}+t_{2}+t_{3}-t_{4}-t_{5}
$$

and

$$
t_{1}=\frac{a_{M-2}+a_{M-1}}{a_{M-2}+a_{M}}, \quad t_{2}=\frac{a_{M-1}+a_{M}}{a_{M-1}+a_{1}}, \quad t_{3}=\frac{a_{M}+a_{1}}{a_{M}+a_{2}}
$$

$$
t_{4}=\frac{a_{M-2}+a_{M-1}}{a_{M-2}+a_{1}}, \quad t_{5}=\frac{a_{M-1}+a_{1}}{a_{M-1}+a_{2}} .
$$

Now $0 \leqq t_{1}-t_{4}$. If $a_{2} \leqq a_{1}$ then $0 \leqq t_{3}-t_{5}$ and since $0 \leqq t_{2}$ we have

$$
0 \leqq E_{M}-E_{M-1} \text {. }
$$

On the other hand if $a_{1}<a_{2}$ then

$$
\begin{aligned}
0 \leqq E_{M}-E_{M-1} & =\left(t_{1}-t_{4}\right)+\frac{a_{M}}{a_{M-1}+a_{1}}+\frac{a_{M}}{a_{M}+a_{2}} \\
& +a_{M-1}\left(\frac{1}{a_{M-1}+a_{1}}-\frac{1}{a_{M-1}+a_{2}}\right)+a_{1}\left(\frac{1}{a_{M}+a_{2}}-\frac{1}{a_{M-1}+a_{2}}\right) .
\end{aligned}
$$

Thus we have proved $4 \leqq \inf E_{M-1} \leqq \inf E_{M}$. To attain the bound 4 let $\left\{a_{i}\right\}=\lambda^{1}, \lambda^{2}, \lambda^{3}, \ldots, \lambda^{M-1}, \lambda^{M-1}$ where $\lambda$ is large.

\section{The Sum in (7)}

The bound $\left[\frac{1}{2}(M+1)\right]$ holds because $F$ has at least one term $\geqq 1$, and $1 \leqq$ the sum of any two adjacent terms of $F$, as is easily verified. Following Diananda's trick (3) we note that

$$
F_{M+2}\left(a_{1}, a_{2}, \ldots, a_{M}, a_{1}, a_{2}\right)=2+F_{M}\left(a_{1}, a_{2}, \ldots, a_{M}\right)
$$

and so inf $F_{M+2} \leqq 2+\inf F_{M}$. Also

$$
\begin{aligned}
F_{M+1}\left(a_{1}, a_{2}, \ldots, a_{r-1}, a_{r}, a_{r}, a_{r+1}, \ldots, a_{M}\right)-F_{M}\left(a_{1}, a_{2}, \ldots, a_{M}\right)-1 \\
=\frac{\left(a_{r-1}-a_{r}\right)\left(a_{r+1}-a_{r}\right)}{2 a_{r}\left(a_{r-1}+a_{r}\right)}
\end{aligned}
$$

which is $\leqq 0$ for some $r$ provided $M$ is odd. Hence inf $F_{M+1} \leqq 1+\inf F_{M}$ 
for $M$ odd. Since inf $F_{R M} \leqq R$ inf $F_{M}$ for any positive integer $R$, it follows that $M^{-1}$ inf $F_{M}$ tends to a limit as $M \rightarrow \infty$. Now

$$
\begin{aligned}
F_{4} & =(a+c)\left\{\frac{1}{a+b}+\frac{1}{c+d}\right\}+(b+d)\left\{\frac{1}{b+c}+\frac{1}{d+a}\right\} \\
& \geqq(a+c)\left\{\frac{4}{(a+b)+(c+d)}\right\}+(b+d)\left\{\frac{4}{(b+c)+(d+a)}\right\}=4,
\end{aligned}
$$

so $M \leqq F_{M}$ for $1 \leqq M \leqq 4$. However $F_{6}=5 \cdot 99902<6$ when

$$
\left\{a_{i}\right\}=381,0,334,29,340,49
$$

and $F_{13}=12.9623<13$ when

$$
\left\{a_{i}\right\}=41,0,28,0,19,4,17,10,18,18,20,29,18 .
$$

It would be interesting to know if inf $F_{M}=M$ for $M=5,7,9,11$, and it seems that this could be determined by means of Nowosad's technique. The lower bound in (7) does not appear to be best possible. When $F_{M}$ is as small as possible the $\left\{a_{i}\right\}$ follow a pattern illustrated by the following example which has $M=110$ and $F_{M}=108 \cdot 735$,

$$
\begin{array}{rrrrrrrrrrrrrrrr}
\left\{a_{i}\right\}= & 0 & 18 & 0 & 16 & 0 & 14 & 0 & 10 & 2 & 12 & 3 & 12 & 4 & 12 & 5 \\
13 & 7 & 14 & 9 & 15 & 10 & 16 & 12 & 17 & 14 & 18 & 16 & 21 & 20 & 23 \\
22 & 26 & 24 & 28 & 27 & 32 & 30 & 36 & 32 & 40 & 36 & 44 & 44 & 52 & 55 \\
64 & 70 & 84 & 94 & 112 & 126 & 154 & 168 & 216 & 222 & 315 & 278 & 478 & 266 & 683 \\
0 & 502 & 0 & 376 & 0 & 286 & 0 & 220 & 0 & 170 & 0 & 132 & 0 & 104 & 0 \\
82 & 0 & 66 & 0 & 56 & 0 & 50 & 0 & 46 & 0 & 42 & 0 & 40 & 0 & 38 \\
0 & 34 & 0 & 32 & 0 & 30 & 0 & 28 & 0 & 26 & 0 & 24 & 0 & 22 & 0 \\
21 & 0 & 20 & 0 & 19 & & & & & & & & &
\end{array}
$$

If $\left\{a_{i}\right\}=a, b, c, \ldots$ then

$$
\frac{\partial F}{\partial c}=\frac{1}{a+b}-\frac{b+d}{(b+c)^{2}}+\frac{d-e}{(c+d)^{2}},
$$

and when $F$ is at its minimum value this derivative is 0 for $c \neq 0$, and is positive for $c=0$. Given any positive $a_{1}, a_{2}, a_{3}, a_{4}$ we can choose $a_{5}$ to make $\frac{\partial F}{\partial a_{3}}=0$, and then $a_{6}$ so that $\frac{\partial F}{\partial a_{4}}=0$, and so on. In this way we can determine

$$
a_{5}, a_{6}, \ldots, a_{M+4} \text { from } a_{1}, a_{2}, a_{3}, a_{4},
$$

but generally we will not have $a_{M+i}=a_{i}$ for $i=1,2,3,4$. However, by iteration we can change $a_{1}, a_{2}, a_{3}, a_{4}$ until $a_{M+i}=a_{i}$ for $i=1,2,3,4$. The corresponding sum $F_{M}$ will be a local minimum. Roughly speaking this was the technique used on a computer to find the examples given here. It is much faster than the method described in (1). 
6. The Inequalities (8) and (9)

Every term of $G_{M}$ is non-positive, and if $\left\{a_{i}\right\}=\lambda^{i}$ then $G_{M} \rightarrow 0$ as $\lambda \rightarrow \infty$. This proves the right hand side of (8). Each term of $H_{M}$ is $\leqq 2 \log 2$, and at least one term is $\leqq 0$, because we must have $a_{i} \leqq a_{i+1}$ for some $i$. Thus $H_{M} \leqq 2(M-1) \log 2$, and to see that this is the best possible bound let $a_{i}=\lambda^{M-i}$ with $\lambda$ large. Since $-\varepsilon^{-1} \leqq x \log _{\varepsilon} x$ for $0 \leqq x$ the left hand sides of $(8)$ and (9) are trivial. It seems that inf $G_{M} \rightarrow-(M-1) \varepsilon^{-1}$ and

$$
\text { inf } H_{M} \rightarrow\left(2 \log _{\varepsilon} 2\right)-(M-1) \varepsilon^{-1} \text { as } M \rightarrow \infty \text {. }
$$

The examples $a_{i}=(\varepsilon-1)^{i-1}$ and $a_{i}=(2 \varepsilon-1)^{i-1}$ respectively support these ideas.

\section{REFERENCES}

(1) D. E. DAykin, Inequalities for functions of a cyclic nature, J. London Math. Soc. (to appear).

(2) P. H. Diananda, A cyclic inequality and an extension of it, Proc. Edinburgh Math. Soc. (2) 13 (1962), 79-84 and 143-152.

(3) P. H. Diananda, On a cyclic sum, Proc. Glasgow Math. Soc. 6 (1963), 11-13.

(4) P. Nowosad, Isoperimetric eigenvalue problems in algebras, Comm. Pure Appl. Math. 21 (1968), 401-465.

(5) A. Zulauf, On a conjecture of L. J. Mordell, Math. Gazette 43 (1959), 42 and 182-184.

DePARTMENT OF MATHEMATICS

UNIVERSITY OF READING 